\title{
Civilisations
}

Revue internationale d'anthropologie et de sciences

humaines

$50 \mid 2002$

Itinéraires belges aux Amériques

\section{Les habitants des rochers ornés du Canada}

\section{Serge Lemaître}

URL : http://journals.openedition.org/civilisations/3385

DOI : 10.4000/civilisations.3385

ISSN : 2032-0442

\section{Éditeur}

Institut de sociologie de l'Université Libre de Bruxelles

\section{Édition imprimée}

Date de publication : 1 décembre 2002

Pagination : 15-30

ISBN : 2-87263-180-1

ISSN : 0009-8140

\section{Référence électronique}

Serge Lemaître, "Les habitants des rochers ornés du Canada ", Civilisations [En ligne], 50 | 2002, mis en ligne le 01 décembre 2004, consulté le 03 mai 2019. URL : http://journals.openedition.org/ civilisations/3385; DOI : 10.4000/civilisations.3385

\section{(c) Tous droits réservés}




\section{LES HABITANTS DES ROCHERS ORNES DU CANADA}

\section{SERGE LEMAITRE}

L'art rupestre du Bouclier canadien est encore fort méconnu malgré près d'un siècle de recherche dans cette région. On compte, à ce jour, plus de 400 sites répertoriés dans les provinces de l'Ontario, du Québec, du Saskatchewan et du Manitoba. La majorité sont des sites à peintures, été réalisées avec de l'hématite, sur des rochers verticaux bordant les lacs ou les rivières. Lors des premières études menées par Selwyn Dewdney dans les années cinquante, plusieurs autochtones ont rapproché les rochers ornés des petits êtres de la mythologie algonquienne : les Maymaygwashiuk. Par la suite, les scientifiques rapporteront $d^{\prime}$ 'autres récits mettant en scène ces lutins d'Amérique. Depuis quelques années, nous menons des recherches sur les sites d'art rupestre du Québec et de l'est ontarien. En 1998, nous avons eu l'occasion de séjourner chez Alex Mathias, un Anishinabek qui vit au lac Obabika (Ontario). Outre l'indication d'un site encore inédit (Lemaitre 2001:6), nous avons pu recueillir un certain nombre de données concernant le paysage sacré des lacs avoisinants. Nous avons ainsi appris l'existence de Maymaygwashiuk sur le lac Diamond où se trouve un des sites rupestres les plus intéressants de la région. Il n'en fallait pas plus pour nous intéresser de plus près à ces êtres fascinants.

Pour servir de base à notre courte étude, nous nous proposons tout $\mathrm{d}^{\prime}$ abord de faire découvrir un récit très complet qui retrace les aventures d'un Amérindien de l'Ontario. Cette histoire a été recueillie par Thor Conway, lors d'une de ses multiples campagnes d'étude des sites rupestres, auprès d'un Ojibwa du nom de Shannon Cryer. Celui-ci lui raconta cette étrange légende ayant pour cadre Matagaming Sagagen, c'est-à-dire l'actuel Horwood lake (Conway 1993:154-157).

Nous analyserons ensuite ce récit afin de comprendre l'identité de ces êtres et les raisons de leurs liens avec les rochers ornés du Canada. 


\section{LE RECIT DE MATAGAMING SAGAGEN}

"Indian people used to see those Maymaygwashiuk fishing with their little nets in front of that rock with the markings on Matagaming Sagagen. As soon as the elves saw you, they would paddle their tiny canoes as fast as they could and disappear into that cliff.

One windy day, an Indian fellow spotted the little people fishing. So he paddled up close to see them. They were hairy all over. But he could hear them speaking the Indian language. All of a sudden, the fairies turned in their canoes and saw the Indian man. The fairies raced toward the cliff with the paintings. But the Indian man paddled hard and kept close behind them.

Suddenly, the wall of paintings opened up in front of the fairies. The little men sped into a deep cave inside the cliff as they often had before. This time, the Indian dug his cedar paddle into the deep lake waters as furiously as he could. He reached the dark opening just as the rock began to close. In an instant, he was inside the fairies' cave.

"You caught us!" the Maymaygwashiuk yelled. They stood on a flat rock shelf that went further back into the cave. Their birchbark canoes were pulled onto the edge of the small bay of lake water that extended into the cave.

"You speak my language," the Indian man said excitedly. "I waited many years to catch you. I want to know how you live. And why do fairies avoid my people?" he asked.

The rock elves laughed. "you tricked us today," they told him; "you caught us and now you can have anything you want." The Indian was so surprised to be talking to this mysterious tribe that he had not taken time to look around their home. As he glanced beyond their miniature camp fires made of twigs, the Indian was startled to find their residence so attractive. Like most of the Matagaming tribe, he thought the little men lived in a dark, damp, hole in the cliff. Instead, the Indian saw a beautiful, perfect image of wilderness. The floor of the cave had the finest yellow sand floor even though there was no sand in that part of the lake.

Old stories raced through the Indian's head. The fairies were respected and feared. They had the power to help Indian people, but fairies also caused trouble. One time some Matagaming Indians were camping near this lake. The parents hung the cradle board up in a tree to protect the baby while they collected limbs for their fire. While the parents spread out to gather more firewood, the Maymaygwashiuk took the baby. The little people ran off with the child still wrapped in blankets and moss safely in the cradle board.

When the baby's parents heard its cries, they chased after the sounds. The little wild men could not get to their canoe fast enough. And they did not want to get caught. After awhile, the Indians found their baby by its cries. The fairies had hung it up on a pine limb on their way to the water. 
Everyone knew that the Maymaygwashiuk stole tools from the Indians. You could never actually see the little people do this. They always hid behind trees or rocks. Sometimes there would be a quick flash of lightning and your axe or knife would be gone. Even on a clear day without clouds, the lightning bolt would strike when they moved. These fairies are a very powerful tribe. They converse with the Thunder people. That is why the Maymaygwashiuk live in caves where there are markings on rock.

Inside, the cave was filled with fine bush food - smoked lake trout, strips of moose meat, woodland caribou stews, snowshoe hares fried with berries, ruffed grouse browning on roasting sticks, fat beaver roasts in bark dishes, everything an Indian could dream for. And one side if the cave covered with painted skin drums, bone-handled flint knives, tightly woven black ash baskets, medicine bags filled with fresh herbs, and many more things. The Indian recognized a few of the things. Some had belonged to his grandparents when he was a boy, and had disappeared long ago. Others looked very old. He had never seen such treasures in his 40 years of life.

"So this is were everything that gets lost ends up," the visitor thought. "I knew that the fairies steal from us, but never dreamed that they lived so well," he said to himself.

"Of course, we do," one of the little wild men answered. "and we hear your thoughts just as we listen to your prayers at the spirit rock. Although we hunt and fish like you, we are influenced by the spirits. We take your messages to Thunder and the other forces that cannot be seen.

The Indian sensed that he was present in a living vision - a place and source of great spiritual power. He accepted the fairy's offer to stay as their guest for one night. It was an evening of good food. He felt as full as the times he sat at a feast to honor bear. ${ }^{2}$. The little wild men drummed until dawn and sang medicine songs so old that the Indian could barely understand the words. When he asked why the song-prayers used such ancient words, the fairy told the Indian that these were the songs he learned in his youth. Then the Indian noticed that all the fairies looked youthful and very old at the same time. With the small fires casting dancing shadows on the smooth walls and ceiling of their home, heartbeat drumming pulsing through the night, and the high-pitched songs echoing across the lake, the Indian received many visions while sitting with the little wild men.

At dawn, the leader of the fairies told the Indian man that it was time for him to rejoin his own nation. As the sunlight spread across the eastern hills, the cave filled with a yellow glow. And the cliff, still transparent from inside, shimmered in the light. The Indian could not believe what he saw. The entire rock wall that he had viewed many times from the outside was clear. Each rock painting hung in the air, reddened to the deepest hue by the arrival of Manidoo Ghesis, the Spirit of the Sun. Matagaming Lake sparkled with globes of fire as each small wave reflected 
the rising sun. And the ancient paintings of stars, suns, caribou, and animal spirits came to life. The broad mouth of the cave formed a clear tapestry of pictograph images.

Low sounds, like a crowd of voices, could be heard around the groups of paintings. As the Indian man walked closer to the magical wall, he understood the sounds. They were the voices of his people! He heard his grandmother praying 30 years ago as she left tobacco at the cliff ${ }^{3}$. And he heard his father and uncles sing the songs of Djiski-Inninik while they painted images fresh from their vision quest. These hunters too had passed to the land beyond the stars long before.

The Indian man stepped into his bark canoe inside the cave near the wall of pictures and prayers. He thanked the little wild men for his visit. No one had ever lived such a full, single evening. The Maymaygwashiuk stood by their tiny canoes as he paddled into the clear wall of paintings. With a few deep strokes, the Indian glided out of the cave. As soon as he turned around to look again at his hosts, he saw only a wall of rock covered with the same paintings, now silent. He thought he heard the sound of tiny drum inside the cliff, but the waves were lapping the sides of his canoe with such force that he could not be sure.

"How will I best use the power of my visit to the little wild men ?" the Indian wondered. He decided to talk with the Elders. The Indian man returned to his camp at the pine-covered point on Matagaming Lake. But something was not right. He beached his canoe where several children were playing. "I have never seen these children" he said. And the summer camp seemed different. Stopping for a moment, he tried to understand what was changed. "the pines!" he yelled. "the pines are so tall now. What is happening ?"

The Indian, who left this village the previous afternoon, was confused. He asked a man standing by a summer lodge, "Who are you ?" The hunter told him his name. "I don't know you." Now the Indian walked over to a group of women who were preparing hides. No one knew him.

Fear rose within the Indian's heart. He asked about his wife and children. The other Indians said they once heard of that family, but that was a long time before. "I remember hearing that story. The husband of that family disappeared one clear day while fishing on the lake" a woman told him. "that was before my time".

Finally, the Indian man spoke to an old, old woman. "Grandmother," he said "do you know of my family?" The old woman looked at him in amazement. "I am your youngest daughter !" she gasped. "You left when I was only a baby. Now I have lived for 80 winters." The Indian man understood. His single evening with the spirits' helpers had lasted a lifetime. He lived a few more years after his return. A night with the fairies had caused a strange event." ${ }^{\prime \prime}$ 
Plusieurs points de ce récit sont particulièrement intéressants et révèlent la manière dont sont vus les Maymaygwashiuk par les autochtones du Bouclier canadien. Nous étudierons tour à tour plusieurs caractéristiques : leur description physique, leur habitat, leur mode de vie, leurs liens avec les esprits et notamment le tonnerre, leurs rôles d'intercesseurs qu'ils occupent entre les esprits et les humains et bien entendu, avec la fin dramatique du récit, la notion du temps.

\section{UN PHYSIQUE INGRAT}

Régulièrement décrits comme des petits personnages d'allure humaine, les Maymaygwashiuk s'en éloignent cependant par différentes caractéristiques : ils n'auraient pas de nez, ou tout du moins pas la partie cartilagineuse (Jones 1981:77) et seraient recouverts de poils, parfois roux (Lambert 1983:x). Il n'est cependant fait aucune mention de cette toison chez les Algonquiens du Nord-ouest (Dewdney 1967:22). Conscients de leur laideur, ils sont timides et honteux de leur apparence comme le montrent plusieurs autres histoires dont ce passage où un pêcheur surprend des Maymaygwashiuk sur le lac : "Les $M$. tombèrent au fond de leur canoë parce qu'ils étaient très honteux de leurs visages. Le vieux pêcheur leur dit de s'asseoir dans le canoë, mais ils répondirent qu'ils ne voulaient pas qu'il voie leurs visages parce qu'ils étaient trop laids. Le vieil homme leur dit qu'il savait à quoi ils ressemblaient parce qu'il les avait déjà vus dans ses rêves. Les $M$. enlevèrent leurs mains de leur visage et s'assirent dans le canoë. Au grand étonnement du vieil homme, ils n'avaient pas de nez » (Ray et Stevens 1971:96)

Ils parlent la langue indienne et sont compréhensibles par les humains, cependant lorsqu'ils chantent des prières, leur vocabulaire est ancien. Nous reviendrons sur ce détail ultérieurement.

\section{UN MONDE EN PARALLELE}

Les Algonquiens divisent traditionnellement leur monde en quatre niveaux (un céleste, un terrestre, un aquatique et un souterrain). Les études ethnographiques nous apprennent que, bien qu'ils ne puissent être directement rattachés aux humains, les Maymaygwashiuk ne sont pas considérés non plus comme des esprits. Pour les Ojibwas, ils sont cependant les habitants du niveau terrestre et sont donc au même titre que les animaux, les arbres, les objets, susceptibles d'être observés. Lors de l'entretien que Shannon a eu avec les Conway, il avait conclu son récit par « so two worlds could exist as one".

Pour nous, Occidentaux du XXI ${ }^{\mathrm{e}}$ siècle, à l'esprit cartésien où tout chose a une place bien définie, ces êtres fabuleux sont de l'ordre des légendes aussi bien que les trolls, farfadets, dragons et autres fantasmagories. Cependant, pour l'esprit amérindien, malgré une forte acculturation, ce type de limites claires n'existe pas. A tout moment, un contact peut être établi avec un esprit (ou manitou) ou un monstre anthropophage tel le Windigo. C'est ainsi, qu'Alex Mathias nous a 
appris que son frère avait eu l'occasion d'observer des Maymaygwashiuk au Diamond lake. Sans mettre une seconde en doute le témoignage de son frère, il insista sur la chance de celui-ci car ces "lutins » se font très rares de nos jours, préférant s'éloigner de toute civilisation (communication personnelle août 1998).

La majorité des rencontres sont très rapides : dès que les petits êtres voient un humain, ils se réfugient à toute allure dans leur rocher. Lors de contacts, ceux-ci se révèlent généralement amicaux (Brown et Brightman 1988:197). Cependant, certains griefs peuvent leur être reprochés. On les accuse notamment de posséder suffisamment de pouvoir pour modifier l'aspect d'un lac en faisant souffler le vent et en provoquant de grosses vagues (Lambert 1983:x). Ils causent d'autres nuisances aux humains et sont souvent décrits comme des chapardeurs. C'est un point sur lequel s'accordent la majorité des sources : ils adorent faire des farces et se nourrissent en volant les poissons pris dans les filets des Amérindiens (Dewdney 1975:12). Ils subtilisent également des objets usuels tels des couteaux, des haches ou des bols. Un cas plus rare qui est décrit ici, c'est le rapt d'enfant : une farce jouée aux parents ou la volonté d'éduquer un enfant à leur manière ?

A la lecture du texte, on découvre également leur mode de vie. A la surprise de notre protagoniste, ces petits êtres vivent de la même manière que lui, voire même mieux. Ils ont tout ce dont un Indien peut rêver et en suffisance, la nourriture ne semble pas manquer et contre toute attente, ils n'habitent pas une caverne humide et sombre. Le sol est recouvert de sable fin et ils ont la lumière du soleil pour les éclairer puisque la paroi de la caverne est transparente de l'intérieur.

Une autre caractéristique, qui fait étrangement défaut ici, est la mention de la matière dans laquelle sont faits leurs canoës. La majorité des autres récits que nous connaissons, indique que leurs embarcations étaient taillées dans la pierre (Lambert 1983: x; Desveaux 1988:265 ; Peyton 1989:4). Ces Maymaygwashiuk, non seulement vivraient à l'intérieur des rochers, mais ils utiliseraient également ce matériel pour la confection de différents objets utilitaires. Ils sont également les détenteurs des médecines minérales ainsi que d'autres produits. Les hommesmédecines ont pour habitude de se fournir dans les anfractuosités et les cavernes (Brown et Brightman 1988:176) comme dans le récit recueilli par Hallowell (1971:59).

\section{DES MESSAGERS DES ESPRITS}

Lors des différentes enquêtes menées par les chercheurs en art rupestre, un grand nombre d'autochtones interrogés lient les peintures à ces petits êtres fabuleux. Un informateur de Dewdney explique que les dessins que nous voyons aujourd'hui sur les rochers représentent des révélations des lutins à l'homme venu leur demander des médecines (Dewdney 1978:115). D'autres disent que des peintures ont été faites sur les rochers pour indiquer où vivent les Maymaygwashiuk, une croix signalerait qu'ils ont quitté l'endroit (Brenner 1994:70). 
Le texte de Shannon nous apprend plusieurs éléments quant au rôle $\mathrm{d}$ 'intercesseur que les Maymaygwashiuk jouent entre les humains et les esprits. Ils entendent les prières qui sont faites au rocher et les transmettent aux esprits du Tonnerre et aux autres manitous, généralement en échange d'offrandes de tabac comme ici, ou parfois de poisson frais (Dewdney 1975:13). Ils interviennent également dans les rites de la tente tremblante ${ }^{5}$ comme conseiller du guérisseur (Hallowell 1971:59). Leur rôle dans les guérisons est aussi perceptible dans ce récit : ils chantent des chants guérisseurs et ils possèdent des sacs médecines pleins d'herbes.

Lors du repas, l'Indien vit une expérience supplémentaire en entrant en transe : les lumières dansantes, le battement du tambour qui se calquent sur celui de son coeur et les chants haut perchés l'entraînent dans le monde des visions. Le son du tambour peut évoquer le bruit sourd du tonnerre qui roule.

Leur lien avec les Oiseaux-Tonnerre est encore accentué dans la manière de procéder pour obtenir une vision. Certains lieux privilégiés pour la quête de vision sont connus et encore fréquentés de nos jours. C'est le cas notamment de Dreamer's Rock sur le Lac Huron. Dan Pine, Ojibwa, a expliqué comment procéder : " tu dois faire un feu à la base de Dreamer's Rock avant d'aller jeûner. Les Petits Hommes Sauvages vivent là. Fais ton feu de l'autre côté du lac à la base du chemin, juste avant qu'il ne monte la pente. Prends des pommes de pins et de l'écorce sèche pour le feu. Pourquoi du pin? C'est l'arbre que le Tonnerre foudroie. Le feu, avec sa fumée, transporte tes souhaits aux Petits Hommes Sauvages. Ils sont les intermédiaires - les intermédiaires entre toi et le Grand Esprit " (Conway 1993:101). Nous voyons avec ce nouvel exemple l'importance de ces petits êtres en tant qu'intercesseurs entre les humains et les manitous. Il faut également remarquer que, comme dans notre récit principal, il existe un lien entre ce petit peuple et le Tonnerre. Ils se déplacent tellement vite que seule une boule de feu est perçue ; l'expression "rapide comme l'éclair» ne pourrait pas mieux s'appliquer. Dans le dernier extrait, ce lien est marqué symboliquement : une essence d'arbre particulière doit être utilisée pour le feu afin de souligner le mimétisme avec le Tonnerre. Le lien se marque d'autant plus que les sites d'art rupestre sont souvent considérés comme les endroits où nichent les Oiseaux-Tonnerre. La plupart des rochers ornés sont, en effet, des falaises parfois très hautes, qui sont vues comme des plates-formes propices à la nidification de ces êtres fantastiques, sortes de rapaces géants, qui par leurs battements d'ailes provoquent le tonnerre et par leurs clignements $d^{\prime}$ yeux projettent des éclairs (Chamberlain 1890:51). Ce rapprochement est renforcée par la présence de grandes coulées minérales blanchâtres, interprétées comme la fiente de ces volatiles, tandis que les veines de quartz que l'on trouve régulièrement dans les granits des rochers peints matérialiseraient l'impact de la foudre. L'exemple des peintures rupestres de la French River, où un oiseau a été peint horizontalement pour qu'il paraisse produire les veines de quartz, réunit l'ensemble des symboles : un rocher vertical, une grande crevasse par où peuvent entrer les Maymaygwashiuk et la matérialisation de l'esprit du Tonnerre sous la forme de l'Oiseau. 


\section{UNE AUTRE DIMENSION TEMPORELLE}

La fin tragique de l'histoire montre clairement un décalage temporel entre les deux mondes. Pour une journée passée auprès des Maymaygwashiuk, près de quatre-vingt années se sont écoulées chez les Humains. Bien qu'ils vivent sur le même plan terrestre et qu'ils fassent de nombreuses incursions dans le monde des Amérindiens, les lutins ne sont pas affectés de la même manière. "Ils paraissent jeunes et très vieux à la fois ». Dans un autre récit, le chef des Maymaygwashiuk exhorte deux jeunes femmes à rester auprès d'eux : "Stay with us and you will always be young and beautiful" (Peyton 1994:5). Leurs chants également utilisent un vocabulaire très ancien que notre protagoniste a du mal à comprendre. On retrouve cette caractéristique dans les chants des hommes-médecines de la confrérie du Midewiwin ${ }^{6}$ ainsi que du Jessakid. Lors des cérémonies de la tente tremblante, les manitous qui visitent le Jessakid parlent tous des langues différentes, néanmoins quand celles-ci sont compréhensibles, elles emploient des phrases archaïques (Preston 1975:73). Les paroles ou tournures de phrases anciennes de ces chants guérisseurs semblent venir de la nuit des temps et se sont transmises de génération en génération.

Notre héros découvre même dans la grotte des objets que ses grand parents croyaient avoir perdus depuis de nombreuses années. Le plus étonnant reste cependant ces chants qu'il entend en s'approchant des images peintes : il reconnaît la voix de son père et d'autres membres de la famille, morts depuis longtemps.

\section{LES SITES D'ART RUPESTRE}

A travers toute l'Amérique du Nord, des petits êtres sont associés aux peintures et gravures rupestres, présentant plus ou moins de similarités avec nos Maymaygwashiuk. Si tous n'ont pas le même physique, ils sont cependant tous des esprits qui aident les chamans dans leur pratique de guérison (Hedges 1993:33-34). Le lien est ici marqué avec leurs rôles d'intercesseurs auprès des Manitous et plus particulièrement le Tonnerre ou l'Oiseau-Tonnerre, manitou protecteur du chaman (Brown et Brightman 1988:175).

Dans notre récit, on découvre que les peintures ont été réalisées par des proches du héros. Son père et ses oncles ont peint le rocher suite à leur quête de vision. Une fois atteint l'âge de l'adolescence, tous les garçons iront, au moment décidé par leur père, s'isoler dans un endroit reculé, propice à leur vision. Ils resteront là quelques jours, sans bouger, le visage, les jambes et les bras noircis de cendre, à jeûner dans l'espoir d'être "visités» par un esprit qui deviendra leur totem et leur protecteur : c'est leur puagan (Johnston 1982:43). J. G. Kohl (1985:143-144) écrit, en 1860, que ces adolescents réalisent des représentations de leur vision sur leurs vêtements mais aussi sur des arbres, des écorces, des tissus et des rochers. Outre cette mention, un informateur Ojibwa de 79 ans signale 
que les peintures se situent à l'endroit où elles furent rêvées par l'artiste (Jones 1981:72). Dans les environs de sites d'art rupestre, on a découvert plusieurs «nids d'Oiseau-Tonnerre ", c'est-à-dire des cercles de pierres, d'un diamètre de plusieurs mètres et d'une profondeur $d$ 'un ou deux mètres, dans lesquels la tradition rapporte que le jeune attendait sa vision (Jones 1979:89-90; Steinbring 1978:11). Bien entendu, nous ne pouvons associer avec certitude ces «nids» aux sites de pictogrammes mais leur proximité est étonnante.

Lorsqu'on demande aux autochtones de quand date la réalisation des peintures, la plupart du temps, ils répondent "a long time ago », parfois ils placent même la réalisation en des temps mythiques très lointains. Les peintures subissent des dégradations à cause de phénomènes naturels (pluie, grands écarts de température...), mais sont aussi protégés par un vernis naturel minéral qui s'est déposé au fil du temps. D'après quelques datations, il semblerait donc que des sites aient pu être réalisés il y a plus de deux mille ans (Arsenault et Gagnon 1993:106). Ces dessins rouges ont pu faire partie du paysage quotidien de ces populations depuis de nombreuses générations, ce qui pourrait expliquer ces datations lointaines et l'écoulement différent du temps pour les Maymaygwashiuk.

CONCLUSION

Bien qu'il ne s'agisse que de légendes, celles-ci nous en apprennent beaucoup sur la manière dont sont perçus les sites rupestres de nos jours. Il en ressort, en effet, plusieurs éléments caractéristiques. Tout d'abord, nous avons vu l'importance du cadre physique : il s'agit d'un grand rocher qui borde le lac et qui possède des crevasses par lesquelles les Maymaygwashiuk peuvent se faufiler dans leurs antres. Ensuite, nous avons constaté l'importance de la sonorité : on croit y entendre des tambours ou des voix. Le texte de Shannon Cryer nous décrit également le lever du soleil sur le rocher faisant danser les pictogrammes. Enfin, nous avons également insisté sur le lien de ces petits êtres avec le Tonnerre. Il serait intéressant d'analyser les sites ornés de l'ensemble du Bouclier canadien en fonction de ces différents éléments. Les recherches que nous avons entreprises depuis quelques années dans la partie orientale, nous ont permis, grâce à ce nouveau regard, de mieux prendre en compte des phénomènes tels que l'acoustique, l'orientation du rocher, l'interaction entre les graphèmes et les particularités de la roche et de comprendre l'élection de certains rochers par rapport à d'autres.

Nous espérons avoir montré par cette courte étude que, si les études ethnographiques ne peuvent nous donner des solutions exactes, tout au moins peuvent-elles nous apprendre à poser les bonnes questions et à regarder non pas avec nos yeux d'occidentaux mais plutôt dans l'esprit des populations qui nous les ont légués. 




Figure 1. photographie du site de Kennedy Island sur la French river. Les veines de quartz sont "produites" par les ailes de l'Oiseau-Tonnerre. (Photographie S. Lemaitre.) 


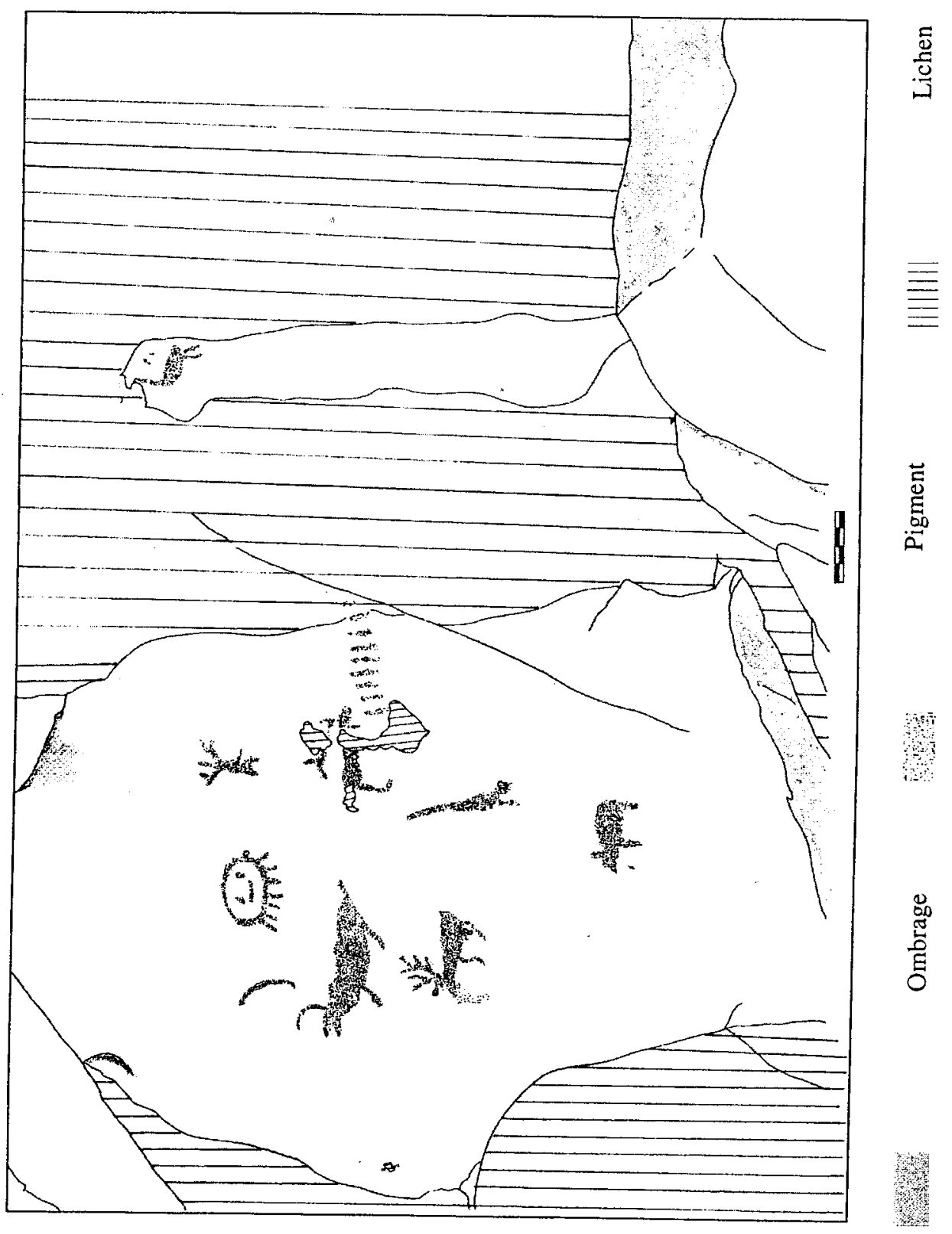

Figure 2. relevé d'un panneau du site de Fairy Point sur Missinaibi Lake (Ontario). Ces graphèmes donnent une idée de ce que notre héros a pu voir lors de son séjour auprès des Maymaygwashiuk. Le toponyme renvoie directement à la présence de ces êtres. Relevé $S$. Lemaitre. 


\section{BIBLIOGRAPHIE}

ARSENAULT, D. et, L. GAGNON,1993, Analyse archéologique et évaluation des conditions de conservation d'un site à pictogrammes de la Zec de Forestville, le site Nisula (DeEh1), Municipalité régionale du Comté de la Haute-Côte-Nord, Québec.

BRENNER, B. 1994 Interpretation of rock art. Manitoba Archaeological Journal 4 (1 et 2): 43-76.

BROWN, J.S.H. et, R. BRIGHTAM, 1988, Orders of the dreamed : G. Nelson on Cree and northern Ojibway religion and myth, 1823. Minnesota Historical Society press, Winnipeg.

CHAMBERLAin, A. F., 1890, The Thunder-bird amongst the Algonkins. American Anthropologist (old series) 3:51-54.

CONWAY, T., 1993, Painted Dreams. Native American Rock art. NorthWord, Hong Kong.

COUTURE, Y. H., 1983, Les Algonquins. Hyperborée, Val d'Or.

DESVEAUX, E., 1988, Sous le signe de l'ours. Mythes et temporalité chez les Ojibwa septentrionaux. M.S.H., Paris.

DEWDNEY, S.

1965, Stone Age paintings. A Heritage Area publication, Toronto.

1978, Aboriginal rock paintings in Manitoba ; a preliminary description of 27 sites east and northeast of the Lake Winnipeg. Miscellaneous Papers in Manitoba Archaeology 8:105-134.

DEWDNEY, S. et, K. E. KIDD, 1967, Indian rock paintings of the Great Lakes, revised edition, Toronto.

GRIM, J.A. , 1993, Chamane, guérisseur de l'âme. Presses Pocket, Paris.

HALLOWELL, A.I., 1971, The role of conjuring in Salteaux Society. Octagon books, New York.

HEDGES, K., 1993, In search of the Little People. Rock Art Papers 10:33-36. 
JONES, L., 1979, Rock art and ethnography in the Nelson River area of Manitoba. Canadian Rock Art Research Assoc. Papers from the 4th biennial conference,1977, Victoria :83-97.

KOHL, J.G., 1985 [1860], Kitch-gami: life among the Lake Superior Ojibway. Minnesota Historiacal Society Press, St Paul.

LAMBERT, P., 1983, Conservation archaeology report: the Northwestern Ontario Rock Art project : the 1982 results. Rapport n², Ministry of Citizenship and Culture, Toronto.

LANDES, R., 1968, Ojibwa religion and the Midewiwin. University of Wisconsin, Madison.

LEMAÎTRE, S., 2001, The rock art sites of Northeastern Ontario revisited. Rapport inédit déposé au Ministry of Citizenship and Culture, Toronto.

PEYTON, J., 1994, The stone canoe and other stories. Mc Donald and Woodward, Tallahassee

PRESTON, R. , 1975, Cree narrative : expressing the personal meanings of events. Canadian Ethnology Service Paper 30. Ottawa, National Museum of Man Mercury series.

RAJNOVICH, G., 1994, Reading rock art. Interpreting the Indian rock paintings of the Canadian Shield. Natural Heritage, Toronto.

RAY, C. et, J. STEVENS, 1971, Sacred Legends of the Sandy Lake Cree. McClelland and Stewart, Toronto.

STEINBRING, J., 1978, Ethnological identification in rock pictography of the Canadian Shield. Miscellaneous Papers in Manitoba Archaeology. 8:3-34.

\section{NOTES}

${ }^{1}$ Plusieurs orthographes coexistent. Memekwesiwuk, Maymaygwaishi, Maymaygwashiuk...

${ }^{2}$ L'ours est un animal très important pour les Ojibwas, il est vénéré et respecté comme s'il était un humain. Le fait de tuer un ours était très souvent accompagné d'un grand festin. (Pour en savoir plus sur la place de l'ours dans la société algonquienne, lire Desveaux $1988: 226-228$; Brown et Brightman $1988: 123)$.

${ }^{3}$ L'offrande de tabac est l'offrande traditionnelle pour entrer en contact avec les esprits. Encore aujourd'hui, il est fréquent de trouver des offrandes de tabac près des sites rupestres. Nous avons ainsi découverts des petits sachets en feuilles de tabac coincés dans des fissures du site du Lac
Mazinaw. 
${ }^{4}$ "Le peuple indien avait l'habitude de voir les Maymaygwashiuk qui pêchaient avec leurs petits filets devant ce rocher orné sur le lac Matagaming. Dès que ces petits êtres vous voient, ils pagaient aussi vite qu'ils peuvent et disparaissent dans la falaise. Par un jour venteux, un Indien aperçut le petit peuple de pêcheurs. Il pagaya pour s'approcher d'eux. Ils étaient recouverts de poils. Mais, il ne pouvait pas les entendre parler la langue indienne. Tout à coup, les lutins se retournèrent dans leurs canoës et virent l'Indien. Ils se précipitèrent vers le rocher aux peintures. Mais l'Indien ramait de toute ses forces et les suivait de très près. Soudain, la paroi ornée s'ouvrit devant les petits êtres et ils s'enfoncèrent dans une grotte profonde comme ils l'avaient déjà fait souvent auparavant. Cette fois, l'indien plongeait sa rame en cèdre dans l'eau du lac avec autant de vigueur qu'il le pouvait. Il atteignit l'ouverture sombre au moment même où la roche commençait à se refermer. En un instant, il fut à l'intérieur de la grotte des lutins.

- "tu nous as attrapés!" crièrent les Maymaygwashiuk. Ils se trouvaient sur une roche plate qui s'étendait dans la grotte. Leurs canoës en écorce étaient tirés sur la rive de la petite baie.

- "vous parlez ma langue », dit l'Indien avec excitation. "Cela fait plusieurs années que j'essaie de vous attraper. Je veux savoir comment vous vivez. Et pourquoi les lutins évitent-ils mon peuple?" - Ils commencèrent à rire. "Tu nous a bien eus aujourd'hui ", lui dirent-ils. "Tu nous a attrapés et maintenant tu peux obtenir tout ce que tu souhaites "

L'Indien était si surpris de parler avec cette tribu mystérieuse qu'il n'avait pas pris le temps de regarder autour de lui. Alors qu'il jetait un coup d'œil à leurs petits feux de camp faits de branchages, l'Indien commença à trouver leur résidence très attachante. Comme la plupart des gens de la tribu Matagaming, il pensait que les petits hommes vivaient dans un trou, noir et humide de la falaise. Au lieu de cela, l'Indien voyait une étendue belle et parfaite. Le sol de la grotte était recouvert d'un sable jaune très fin, alors qu'il n'y avait pas de sable dans cette partie du lac.

De vieilles histoires lui revinrent en mémoire. Les lutins étaient respectés et craints. Ils avaient le pouvoir d'aider le peuple indien, mais ils provoquaient également des désagréments. Une fois, alors que quelques Indiens Matagaming campaient près de ce lac, un couple pendit le porte-bébé dans un arbre pour protéger leur enfant pendant qu'ils ramassaient des branches pour leur feu. Une fois, les parents éloignés pour récolter plus de bois, les Maymaygwashiuk emportèrent le bébé. Le petit peuple a couru avec l'enfant encore emballé dans les couvertures et la mousse du porte-bébé. Quand les parents entendirent les cris de leur enfant, ils se mirent à courir vers les appels. Les petits hommes ne pourraient pas atteindre assez vite leur canoë. Et ils ne voulaient pas se faire attraper. Après un moment, les Indiens découvrirent leur bébé grâce à ses pleurs. Les lutins l'avaient attaché à une branche de pin sur le trajet.

Chacun sait que les Maymaygwashiuk volent les outils des Indiens. Vous ne parvenez jamais à les voir faire cela. Ils sont toujours cachés derrière les arbres ou les rochers. Parfois, il y a un rapide éclair de lumière et votre hache ou votre couteau a disparu. Même par temps clair et sans nuage, une boule d'éclair peut jaillir quand ils se déplacent. Ces lutins forment une tribu très puissante. Ils parlent avec le peuple du tonnerre. C'est pourquoi les Maymaygwashiuk vivent dans les grottes où le rocher est orné.

A l'intérieur, la grotte était remplie de nourriture fine - de la truite fumée, des lanières de viande d'orignal, du ragoût de caribou, du lièvre "snowshoe " grillé avec des baies, des brochettes de grouse à collerette, du castor gras cuisant dans des plats en écorce, tout ce dont un Indien pouvait rêver. Un des côtés de la grotte était recouvert avec des tambours aux peaux peintes, des couteaux de pierre avec des manches en os, des paniers noirs tressés finement, des sacs-médecine remplis d'herbes fraîches, et bien d'autres choses. L'Indien reconnut une partie des objets. Certains avaient appartenu à ses grands-parents alors qu'il était enfant, et avaient disparu depuis bien longtemps. D'autres objets paraissaient très anciens. Il n'avait jamais vu un tel trésor en quarante ans de vie.

- "Ainsi c'est ici qu'aboutissent tous les objets perdus", pensa-t-il. « Je savais que les lutins nous volaient, mais je n'aurais jamais imaginé qu'ils vivaient si bien. "

- "c'est pourtant le cas" répondit un des petits hommes. "Et nous entendons vos pensées comme nous entendons vos prières à ce rocher sacré. Bien que nous chassions et pêchions tout comme vous, nous sommes influencés par les esprits. Nous apportons vos messages au Tonnerre et les autres forces qui ne peuvent être vues."

L'Indien sentait qu'il vivait une véritable expérience de vision. Il accepta l'offre des lutins d'être leur invité pour une nuit. Ce fut une soirée de festin. Il se sentait rassasié comme la fois où il avait assisté au festin en l'honneur de l'ours ${ }^{3}$. Les petits hommes sauvages ont frappèrent le tambour jusqu'à l'aube et chantèrent des chants de guérison si anciens que l'Indien pouvait à peine en comprendre les paroles. Lorsqu'il demanda pourquoi les chants de prière utilisaient des mots anciens, le lutin lui 
répondit que c'étaient les chants qu'il avait appris dans sa jeunesse. A ce moment, l'Indien nota que tous les lutins paraissaient à la fois jeunes et très vieux. Grâce aux petits feux qui faisaient des ombres dansantes sur les douces parois de leur maison, aux tambours reproduisant les battements de cour tout au long de la nuit, et aux chants haut perchés faisant écho à travers le lac, l'Indien reçut beaucoup de visions.

A l'aube, le chef des lutins dit à l'Indien qu'il était temps pour lui de regagner son propre peuple. Alors que la lumière du soleil se répandait à travers les montagnes de l'est, la grotte se remplit d'une lumière jaune. La falaise, toujours transparente depuis l'intérieur, chatoyait dans la lumière. L'Indien ne pouvait en croire ses yeux. Le rocher qu'il avait vu si souvent de l'extérieur était tout entier transparent. Toutes les peintures flottaient dans les airs, rougissant dans des tons très profonds avec l'arrivée de Manidoo Ghesis, l'esprit du Soleil. Le Matagaming lake scintillait de boules de feu chaque fois que les vagues réfléchissaient le soleil. Et les anciennes peintures d'étoiles, de soleil, de caribou et d'esprits des animaux devenaient vivantes. La large bouche de la grotte formait une tapisserie transparente de pictogrammes.

Des bruits sourds, comme les voix d'une foule, résonnaient à proximité des peintures rupestres. Comme l'Indien s'approchait de la paroi magique, il en saisit les sons. C'était les voix de son peuple! Il entendait sa grand-mère prier alors qu'elle faisait une offrande de tabac il y a de cela trente ans. Et il entendit son père et ses oncles chanter les chants de Djiski-Inninik pendant qu'ils peignaient les images reçues pendant leur quête de vision. Ces chasseurs étaient passés aussi au pays derrière les Étoiles, il y avait de cela longtemps.

L'Indien embarqua dans son canoë d'écorce à l'intérieur de la grotte, près de la paroi de peintures et de prières. Il remercia les petits êtres pour leur accueil. Personne n'avait jamais vécu une telle nuit. Les Maymaygwashiuk se tenaient près de leurs petits canoës tandis qu'il traversait en pagayant la paroi ornée et transparente. En quelques coups de rame, l'Indien glissa hors de la grotte. Lorsqu'il se retourna pour regarder une dernière fois ses hôtes, il vit seulement une paroi rocheuse couverte des mêmes pictogrammes, à présent silencieux. Il lui sembla avoir entendu le son d'un petit tambour à l'intérieur de la falaise, mais les vagues battaient les côtés de son canoë avec une telle force qu'il n'était pas sûr.

"Comment puis-je utiliser au mieux ma visite chez ces petits hommes sauvages ?" se demanda l'Indien. Il décida d'en parler aux aînés. L'Indien retourna à son camp à la pointe du lac Matagaming qui est recouverte de pins. Mais quelque chose n'allait pas. Il accosta à l'endroit où plusieurs enfants étaient en train de jouer. "Je n'ai jamais vu ces enfants" se dit-il. Et le camp d'été semblait différent. S'arrêtant un moment, il essaya de comprendre ce qui avait changé. "Les pins !" s'exclama-t-il. " Les pins sont si grands maintenant. Qu'est-il arrivé ? "

L'Indien, qui avait quitté son village la veille dans l'après-midi, était troublé. Il demanda à un homme se tenant près d'une loge d'été : "Qui es-tu?". Le chasseur lui dit son nom. "Je ne te connais pas. " répondit l'Indien. Ensuite il se dirigea vers un groupe de femmes qui préparaient les peaux. Aucune ne le connaissait. La peur monta dans le cœur de l'Indien. Il s'enquit de sa femme et ses enfants. Les autres Indiens répondirent qu'ils avaient entendu parler de cette famille, mais c'était il y a très longtemps. "Je me rappelle avoir entendu cette histoire. Le mari de la famille disparut par un beau jour alors qu'il pêchait sur le lac » lui dit une femme, "c'était longtemps avant moin

Finalement, $1^{\prime}$ Indien parla à une très vieille femme. "Grand-mère " dit-il « connais-tu ma famille?". La vieille femme le regarda avec étonnement. "Je suis ta plus jeune fille!" dit-elle le souffle coupé. «Tu es parti quand j'étais seulement un bébé. Maintenant j'ai passé 80 hivers. » L'Indien comprit. Son unique nuit avec les assistants des esprits avait duré une vie entière. Il vécut quelques années encore après son retour. Une nuit avec les lutins avait provoqué un événement étrange. » (Traduction de l'auteur).

${ }^{5} \mathrm{Ce}$ rite consiste à construire une sorte de cylindre en écorce dans lequel le chaman prendra place. Il invoquera alors les manitous qui viendront l'aider. Lorsque ceux-ci s'engouffrent dans la tente, celle-ci se met à trembler.

${ }^{6}$ le Midewiwin est aussi appelé la Grande Société de Médecine. Elle comporte plusieurs niveaux d'initiation où le participant apprend l'histoire du groupe, la mythologie ainsi que des techniques de guérison. Leurs chants sont gravés sur des écorces de bouleau (Landes 1961 ; Grim 1993:222-235). 